\title{
The Need of Big Data Analytics in Healthcare
}

\author{
Serik Smagulov, \\ Viktoriya Smagulova MD
}

\begin{abstract}
This article addresses the need for big data analytics in the healthcare system. The use of big data demonstrates interesting perspectives for improving health outcomes and cost control. The author noted that the use of big data analytics in health care has many positive results. With the introduction of digital technology, it has become possible not only to collect such data but also to convert it into important information that can later be used to provide better service. The advantages of using big data for online reporting and business intelligence, for tracking various user statistics and vital functions are considered. It noted the importance of collecting and processing data for continuous monitoring of vital body functions so that it is possible to identify potential health problems and assist before the situation worsens. The author also identifies the problem of confidentiality of the collected and analyzed data. At the same time, the positive aspects of this process are noted: the introduction of big data in healthcare significantly increases the confidentiality of data and patient safety. This is due to the fact that the information is stored in data centers with different levels of security. In general, according to the author, big data is a new way to overcome existing problems in world medicine.
\end{abstract}

Keywords: Big data, Business Analytics, Internet of Things, healthcare.

Big data has long been accessible in various industries, but their application in health care is still in its infancy. The use of big data demonstrates interesting perspectives for improving health outcomes and cost control.

Big data in the healthcare industry is changing approaches to treating patients and doctors $[1 ; 2]$.

The more data involved, the more effective health care can be.

The use of big data analytics in healthcare has many positive results. Health care uses specific data about the health of the population (or a particular person). In this regard, the analysis of big data allows you to identify signs of serious diseases as they arise.

The treatment of any disease at an early stage is much easier and cheaper. Using medical data analysis for prevention is better than cure, and the ability to create a comprehensive picture of the patient will allow insurance companies to provide an individual package of services.

Not automated collection of vast amounts of data for medical use is expensive and time-consuming. With the introduction of digital technology, it has become possible not only 
to collect such data but also to convert it into relevant information that can later be used to provide better service. This is the purpose of analyzing health data.

The need for big data is due to rising costs in healthcare in many countries [3]. But there are many obstacles to the widespread dissemination of medical data [4].

One of the most significant barriers to the use of big data in medicine is the way medical information is distributed across many sources. Integration of these data sources requires the development of a new infrastructure in which all data providers will cooperate with each other.

The introduction of new software for online reporting and business intelligence is equally important [5].

The goal of business intelligence in health care is to help doctors make decisions based on the data obtained in a short time and improve patient care.

Big data can drastically modernize the health sector.

Big data and analytics, along with the Internet of Things (IoT), is revolutionizing a way to track various user statistics and vital functions [6].

In addition to the primary wearable devices that can monitor the patient's sleep, heart rate, exercise, etc., there are new medical innovations that help control the patient's blood pressure, blood glucose and much more.

Continuous monitoring of vital body functions, as well as data collection from sensors, will help identify potential health problems and provide assistance until the situation worsens.

Big data helps reduce the cost of medical institutions $[7 ; 8]$. They become an excellent way to save costs for hospitals with large numbers of medical personnel to help with its distribution.

In fact, big data helps maximize investment. The use of medical trackers allows patients to stay at home under 24-hour remote medical supervision, which in turn reduces the funds of insurance companies for their inpatient stay. Predictive analytics based on Big Data also helps reduce costs by reducing the frequency of re-admissions. 
Another advantage of Big Data is to help high-risk patients [9; 10]. The ideal option is to digitize all hospital data that can be accessed. This will help identify patients who go to the hospital repeatedly and identify their chronic problems. Such an understanding will help to take better care of such patients and provide insight into corrective measures to reduce their inpatient stay.

Big Data helps to prevent human errors. Often there are cases when experts prescribe the wrong medicine or send a prescription by mistake. It is possible to reduce the risk of such errors by using big data to analyze user data and prescribed medications. Such software can be an excellent tool for doctors who serve many patients per day.

Big data can be a significant advantage for the development of science and technology. In the health sector, some programs can be used to view multiple data for a few seconds in order to find solutions for various diseases.

Big Data can improve the quality of services. Predictive analytics helps to get more accurate results. Moreover, algorithms can provide personalized treatment based on existing information.

Personalization includes comparing drugs with a person's genetic characteristics and developing them based on various factors such as the environment and lifestyle.

In addition, patient data will not be analyzed in isolation. They will be considered along with other cases that allow you to create templates for better analysis. Thus, doctors get valuable predictive modeling with data on how to cure patients with the same conditions.

There are already several completed projects in the field of Big Data. For example, Apple and IBM have developed a big data platform for exploring new biometric data and activity in real time. Users can track health-related data, and the system will offer appropriate treatment for them. In addition, these medical tips will be based on human habits, age, past conditions, etc.

The primary goal of such a platform and healthcare devices is to give people the opportunity to get professional advice remotely. Information from smart devices allows doctors to keep in touch with their patients, track their condition and set an automatic reminder about taking medication. 
Another need to use big data in healthcare is related to informed strategic planning.

The use of big data in health care allows for strategic planning due to a better understanding of people's motivation. You can analyze the results of checks among people in different demographic groups and determine, for example, which factors prevent people from starting early treatment.

As an example, consider the activities of the University of Florida, where Google Maps and free public health data were used to prepare maps reflecting various problems, such as population growth and chronic diseases.

Subsequently, scientists compared these data with the availability of medical services in different areas. The findings allowed them to review their work strategy and add more care units to the most problematic areas.

Embedding big data in healthcare significantly increases data privacy and patient safety. The main reason is that their information is stored in data centers with different levels of security. Moreover, data comes from different places, which carries additional risks.

A cloud solution that uses big data helps strengthen security to meet HIPAA requirements, which is unique to the healthcare industry. Also, it is a cost-effective solution.

Many companies use a hybrid approach to their data storage strategy with flexible functions. However, it should be borne in mind that all systems can interact and exchange data with other segments of the company.

Lack of control may allow fraudsters to hack the system. In this regard, there may be enormous losses for the company.

In general, big data is a new way to overcome existing problems in world medicine, such as low budget and overworked medical workers. When doctors have all the necessary information in one place, you can achieve excellent personalization and offer treatment that is suitable for a specific person.

\section{References}

1. Viceconti M, Hunter P, Hose R. Big data, big knowledge: big data for personalized healthcare. IEEE J Biomed Health Inform 2015;19:1209-15. 
2. Kankanhalli A, Hahn J, Tan S, Gao G. Big data and analytics in healthcare: introduction to the special section. Inform Syst Front 2016;18:233-5.

3. Raghupathi W, Raghupathi V. Big data analytics in healthcare: promise and potential. Health Inform Sci Syst 2014;2:3.

4. Borne K. Top 10 big data challenges - a serious look at 10 big data V's. MAPR, 2014:NO4, 80.

5. El-Gayar O, Timsina P. Opportunities for business intelligence and big data analytics in evidence based medicine. In: System Sciences (HICSS), 2014 47th Hawaii international conference on 2014:749-57.

6. Yuehong YIN, Yan Zeng, Xing Chen, Yuanjie Fan. The internet of things in healthcare: An overview. // Journal of Industrial Information Integration. Volume 1, March 2016, Pages 3-13.

7. Tan SL, Gao G, Koch S. Big data and analytics in healthcare. Methods Inf Med 2015;54:546-7

8. Hermon R, Williams PA. Big data in healthcare: what is it used for? In: Australian Ehealth Informatics and Security Conference. 2014:40-9.

9. Andreu-Perez J, Poon CC, Merrifield RD, Wong ST, Yang GZ. Big data for health. IEEE J Biomed Health Inform 2015;19:1193-1208.

10. Wang Y, Kung LA, Wang WY, Cegielski CG. An integrated big data analyticsenabled transformation model: application to health care. Inf Manag 2017;55:6479. 